\title{
OPEN The expression and role of TRPV2 in esophageal squamous cell carcinoma
}

\author{
Michihiro Kudou ${ }^{1,6}$, Atsushi Shiozaki ${ }^{1,6^{*}}$, Yuzo Yamazato ${ }^{1,6}$, Keita Katsurahara ${ }^{1}$, \\ Toshiyuki Kosuga ${ }^{1}$, Katsutoshi Shoda ${ }^{1}$, Tomohiro Arita ${ }^{1}$, Hirotaka Konishi ${ }^{1}$, Shuhei Komatsu ${ }^{1}$, \\ Takeshi Kubota ${ }^{1}$, Hitoshi Fujiwara ${ }^{1}$, Kazuma Okamoto ${ }^{1}$, Mitsuo Kishimoto ${ }^{2}$, Eiichi Konishi ${ }^{2}$, \\ Yoshinori Marunaka ${ }^{3,4,5}$ \& Eigo Otsuji ${ }^{1}$
}

Background: Transient receptor potential vanilloid 2 (TRPV2) was recently shown to be involved in migrant potentials. The present study aimed to investigate its role in esophageal squamous cell carcinoma (ESCC). Methods: Knockdown experiments were conducted using TRPV2 siRNA in human ESCC cell lines, and anti-tumor effects were analyzed. The gene expression profiles of cells were analyzed using a microarray method. An immunohistochemical staining was performed on 62 primary tumor samples. Results: TRPV2 overexpression was observed in TE15 and KYSE170 cells. TRPV2 depletion suppressed proliferation, cell cycle progression, and invasion/migration ability, and induced apoptosis. A pathway analysis of microarray data showed that TRPV2 depletion down-regulated WNT/ $\beta$-catenin signaling-related genes and basal cell carcinoma signaling-related genes. The suppression of tumor functions, such as proliferation, invasion, and angiogenesis, was predicted in the ontology analysis. Immunohistochemical analysis revealed a correlation between strong TRPV2 expression and a poor prognosis in ESCC patients. Conclusion: The present results suggest that TRPV2 regulates cancer progression by affecting WNT/3-catenin or basal cell carcinoma signaling, and that TRPV2 strong expression is associated with a worse prognosis in ESCC patients. These results provide an insight into the role of TRPV2 as a novel therapeutic target or biomarker for ESCC.

Transient receptor potential vanilloid (TRPV) channels have been identified as one of the molecules activated by capsaicin, a vanilloid-like molecule. TRPV1 was initially reported as a membrane protein triggered by heat or pain stimuli, VR1 in $1997^{1}$. Five additional molecules of the subfamily were subsequently cloned and named TRPV2, TRPV3, TRPV4, TRPV5, and TRPV $6^{2-6}$. Although all members of the TRPV subfamily were initially presumed to have similar functions, such as heat sensors, extensive physiological studies later revealed that TRPV2-6 did not all respond to temperature stimuli. Moreover, TRPV1-4 channels have non-selective cation-conducting pores, while the pores of TRPV5 and TRPV6 are highly calcium selective ${ }^{7}$. Among members of the TRPV subfamily, TRPV2 is expressed in many organs, such as the digestive tract, pancreas, and liver; furthermore, TRPV2 has been implicated in bowel inflammation, intestinal peristalsis, and the autocrine effects of insulin on pancreatic $\beta$-cells ${ }^{8-10}$. Therefore, TRPV 2 may be a therapeutic target of digestive diseases.

Previous studies reported that TRPV2 was involved in cancer progression, migration, and invasion as well as in the therapeutic effects of anticancer drugs. Gambade et al. showed that the activation of TPRV2 regulated cancer migration via calcium entry ${ }^{11}$. Similarly, the involvement of TRPV2 in migration and invasion was demonstrated in bladder and prostate cancers ${ }^{12,13}$. TRPV2 was identified as a poor prognostic marker in gastric cancer and, in contrast, a good prognostic marker in breast cancer ${ }^{14,15}$. Liberati et al. interpreted this discrepancy as TRPV2 playing a major role in initial events that parallel the transformation of a single normal stem/

\footnotetext{
${ }^{1}$ Division of Digestive Surgery, Department of Surgery, Kyoto Prefectural University of Medicine, Kyoto, 602-8566, Japan. ${ }^{2}$ Department of Pathology, Kyoto Prefectural University of Medicine, Kyoto, 602-8566, Japan. ${ }^{3}$ Department of Molecular Cell Physiology, Graduate School of Medical Science, Kyoto Prefectural University of Medicine, Kyoto, 602-8566, Japan. ${ }^{4}$ Research Center for Drug Discovery and Pharmaceutical Development Science, Research Organization of Science and Technology, Ritsumeikan University, Kusatsu, 525-8577, Japan. ${ }^{5}$ Research Institute for Clinical Physiology, Kyoto Industrial Health Association, Kyoto, 604-8472, Japan. ${ }^{6}$ These authors contributed equally: Michihiro Kudou, Atsushi Shiozaki and Yuzo Yamazato. *email: shiozaki@koto.kpu-m.ac.jp
} 
progenitor cell into a tumor cell, while the expression of TRPV2 was modulated during carcinogenesis that followed long-term and continuous exposure to different carcinogenetic agents, resulting in tumor growth, progression, and the selection of more aggressive tumor clones ${ }^{16}$. We demonstrated that TRPV2 was overexpressed in cancer stem cells derived from ESCC, and suggested the potential of tranilast, a TRPV2-specific inhibitor, as a therapeutic agent for cancer stem cells ${ }^{17}$. Although alterations in TRPV2 expression in cancer cells or the involvement of TRPV2 in cancer functions have been reported in several types of carcinomas including esophageal squamous cell carcinoma (ESCC) ${ }^{18}$, the oncological and physiological roles of TRPV2 in ESCC have remained unclear. The elucidation of these roles is needed to develop TRPV2-targeted therapies for ESCC.

Hence, we investigated the role of TPRV2 in ESCC using a cancer function assay with the knockdown of TRPV2 by siRNA, microarray, pathway, and gene ontology analyses. The significance of TRPV2 expression in ESCC samples was then evaluated by immunohistochemical staining. The present study aimed to clarify the role of TRPV2 and its clinical significance in ESCC.

\section{Materials and Methods}

All methods, especially the experiments using human samples, were carried out in accordance with relevant guidelines $^{19}$.

Ethical approval and consent to participate. This study was approved by the Research Ethics Committee of the Kyoto Prefectural University of Medicine (No. ERB-C-1178). Comprehensive informed consent for use of clinical data and samples was obtained from all eligible patients.

Cell culture, antibodies, and other materials. The human ESCC cell line TE5, TE8, TE19m and TE15 was obtained from the Cell Resource Centre for Biomedical Research at the Institute of Development, Aging, and Cancer (Tohoku University, Sendai, Japan). The human ESCC cell lineLYSE150 and KYSE170 was obtained from the Japanese Collection of Research Bioresources Cell Bank (Osaka, Japan). The cells were cultivated using our previously reported protocols ${ }^{20}$. These cell lines were grown in RPMI-1640 medium (Nacalai Tesque, Kyoto, Japan) supplemented with $100 \mathrm{U} / \mathrm{mL}$ penicillin, $100 \mu \mathrm{g} / \mathrm{mL}$ streptomycin, and $10 \%$ fetal bovine serum (FBS). Cells were cultured in flasks and dishes in a humidified incubator at $37^{\circ} \mathrm{C}$ in $5 \% \mathrm{CO}_{2}$ in air. A rabbit monoclonal anti-TRPV2 antibody was used for the immunohistochemical analysis, and a protein assay was obtained from Santa Cruz Biotechnology (Santa Cruz, CA, USA). The following antibodies were used in the Western blotting analysis: a rabbit monoclonal anti-caspase 3 antibody and rabbit monoclonal anti-cleaved caspase 3 antibody, which were purchased from Cell Signaling Technology (Beverly, MA). A mouse monoclonal anti- $\beta$-actin antibody was purchased from Sigma-Aldrich (St. Louis, MO, USA).

Western blotting. Western blotting was performed using our previously reported protocols ${ }^{20}$. Briefly, cells were harvested in M-PER lysis buffer (Pierce, Rockford, IL) supplemented with protease inhibitors (Pierce, Rockford, IL). Protein concentrations were measured with a modified Bradford assay (Bio-Rad, Hercules, CA). Cell lysates containing equal amounts of total protein were separated by SDS-PAGE and then transferred onto PVDF membranes (GE Healthcare, Piscataway, NJ). These membranes were then probed with the indicated antibodies, and proteins were detected using an ECL Plus Western Blotting Detection System (GE Healthcare, Piscataway, NJ).

Small interfering RNA (siRNA) transfection. SiRNA transfection was carried out using our previously reported protocols ${ }^{20}$. Cells were transfected with $20 \mathrm{nmol} / \mathrm{L}$ TRPV2 siRNA (Stealth RNAi siRNA \#HSS122144, Invitrogen, Carlsbad, CA) using Lipofectamine RNAiMAX reagent (Invitrogen) in accordance with the manufacturer's instructions. Medium containing siRNA was replaced with fresh medium after $24 \mathrm{~h}$. Control siRNA (Stealth RNAi siRNA Negative Control; Invitrogen) was used as a negative control.

Cell proliferation assay. Cell proliferation assay was similarly performed using our previously reported protocols ${ }^{20}$.

Cells were seeded on 6 -well plates at a density of $1.5 \times 10^{5}$ cells per well for TE15 and $0.4 \times 10^{5}$ cells per well for KYSE170, and incubated at $37^{\circ} \mathrm{C}$ with $5 \% \mathrm{CO}_{2}$. siRNA was transfected $24 \mathrm{~h}$ after cells had been seeded. Cells were detached from flasks with trypsin-EDTA 48 and $72 \mathrm{~h}$ after siRNA transfection and were counted using a hemocytometer.

Cell cycle analysis. Cell cycle progression was observed using our previously reported protocols ${ }^{20}$. In TRPV2 knockdown experiments, cell cycle progression was evaluated $72 \mathrm{~h}$ after siRNA transfection using FACS. Briefly, cells were treated with Triton X-100, and cell nuclei were stained with PI RNase staining buffer (Becton-Dickinson Biosciences, San Jose, CA, USA). The DNA content was then measured using a Becton-Dickinson Accuri C6 (Becton-Dickinson Biosciences). At least 10,000 cells were counted, and BD Accuri C6 software was used to analyze the cell cycle distribution.

Analysis of apoptotic cells. Cell apoptosis was evaluated using our previously reported protocols ${ }^{20}$. Cells were harvested $72 \mathrm{~h}$ after siRNA transfection and stained with fluorescein isothiocyanate (FITC)- conjugated Annexin V and propidium iodide (PI) using an Annexin V-FITC kit (Beckman Coulter, Brea, CA) in accordance with the manufacturer's protocol. The proportion of apoptotic cells was analyzed by fluorescence-activated cell scoring (FACS) using a BD Accuri C6 (BD Biosciences).

Real-time reverse transcription-polymerase chain reaction (RT-PCR). Gene expression was assessed by our previously reported RT-PCT method ${ }^{20}$. Total RNA was extracted using a RNeasy kit (Qiagen, 


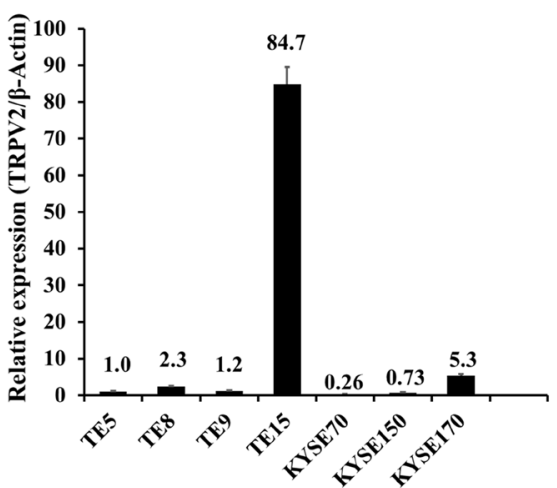

TE15
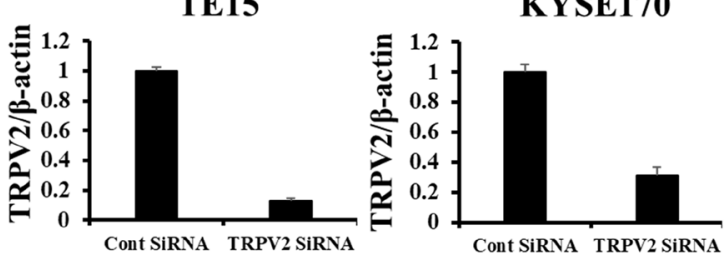

TRPV2

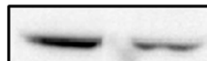

$\beta$-actin

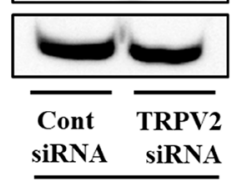

TE15

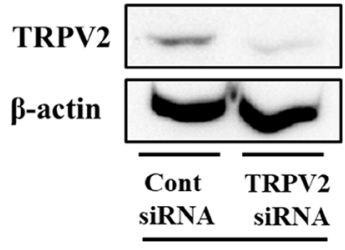

KYSE170

B

$\mathbf{E}$

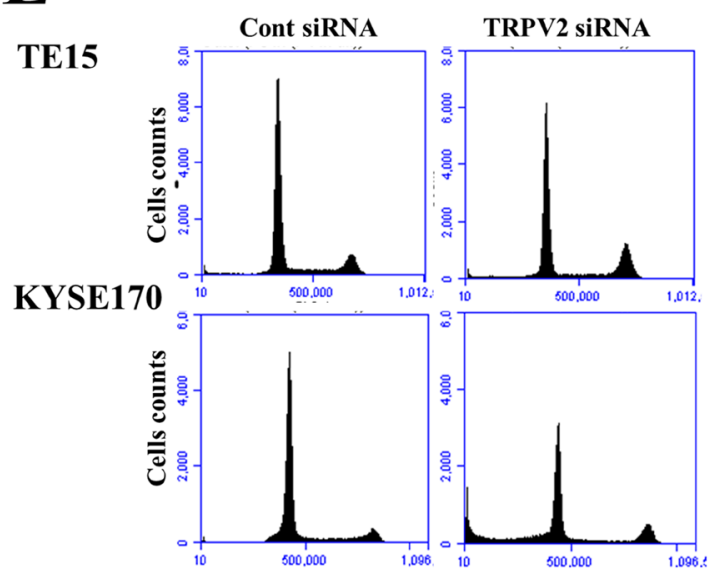

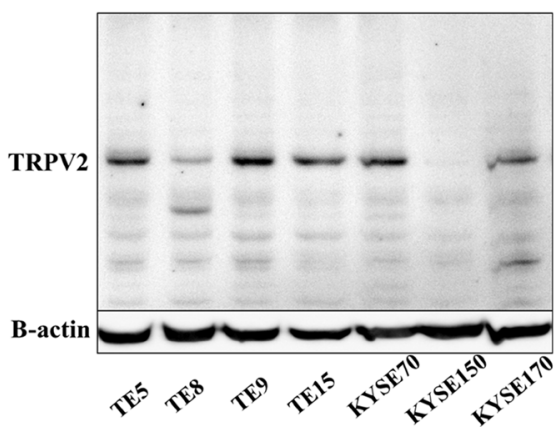

D

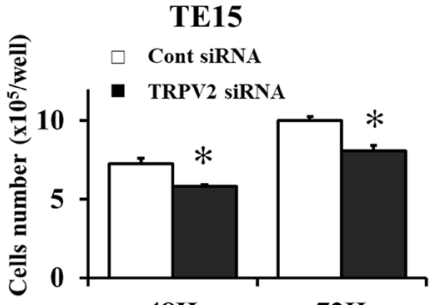

$48 \mathrm{H}$

KYSE170
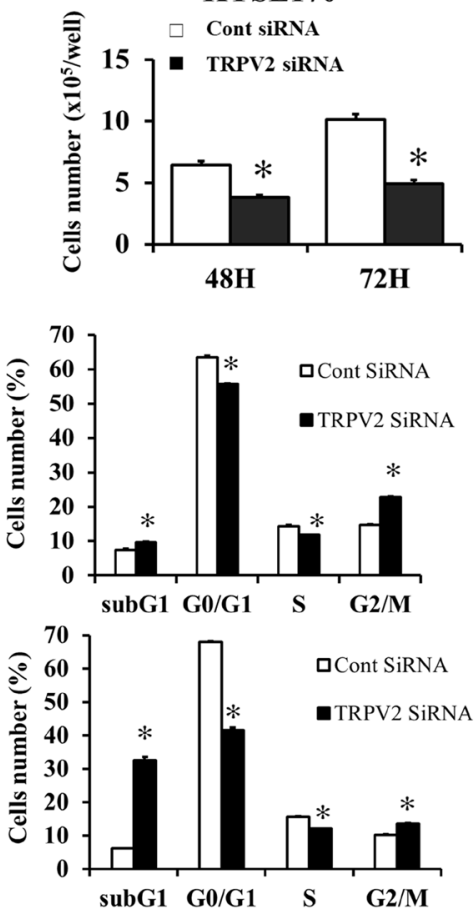

Figure 1. TRPV2 controls the proliferation and cell cycle progression of ESCC cells, (A) TRPV2 mRNA expression was analyzed in 7 ESCC cell lines. Quantitative RT-PCR showed that TRPV2 was strongly expressed in TE15, and more strongly expressed in KYSE170 than in the other ESCC cell lines. (B) TRPV2 protein expression of ESCC cell lines was evaluated by western blotting. Upper panel showed full-length gels of TRPV2, and lower panel indicated the cropped image of same gels for evaluating $\beta$-actin expression (full-length image: Supplementary Fig. 3). TRPV2 expression in TE15 and KYSE170 was similar in Western blotting. (C) TRPV2 expression of ESCC cell lines transfected with control and TRPV2 siRNA was evaluated by quantitative RT-PCR and western blotting. The blotting figure showed the cropped image of TRPV2 or $\beta$-Actin bands on same gels (full-length image: Supplementary Fig. 4). TRPV2 siRNA effectively reduced TRPV2 mRNA and protein levels in TE15 and KYSE170 cells. Mean \pm SEM. $\mathrm{n}=3 .{ }^{*} p<0.05$ (significantly different from control siRNA). (D) The down-regulation of TRPV2 inhibited the proliferation of TE15 and KYSE170 cells. The number of cells was counted 48 and $72 \mathrm{~h}$ after siRNA transfection. Mean \pm SEM. $\mathrm{n}=4$. $* p<0.05$ (significantly different from control siRNA). (E) The down-regulation of TRPV2 partially reduced cell cycle progression from the G1 to $S$ phase in TE15 and KYSE170 cells. The cell population of sub-G1 increased in TPRV2-depleted TE15 and KYSE170 cells. Cells transfected with control or NIS siRNA were stained with propidium iodide (PI) and analyzed by flow cytometry. Mean \pm SEM. $\mathrm{n}=3$. ${ }^{*} p<0.05$ (significantly different from control siRNA). 
A
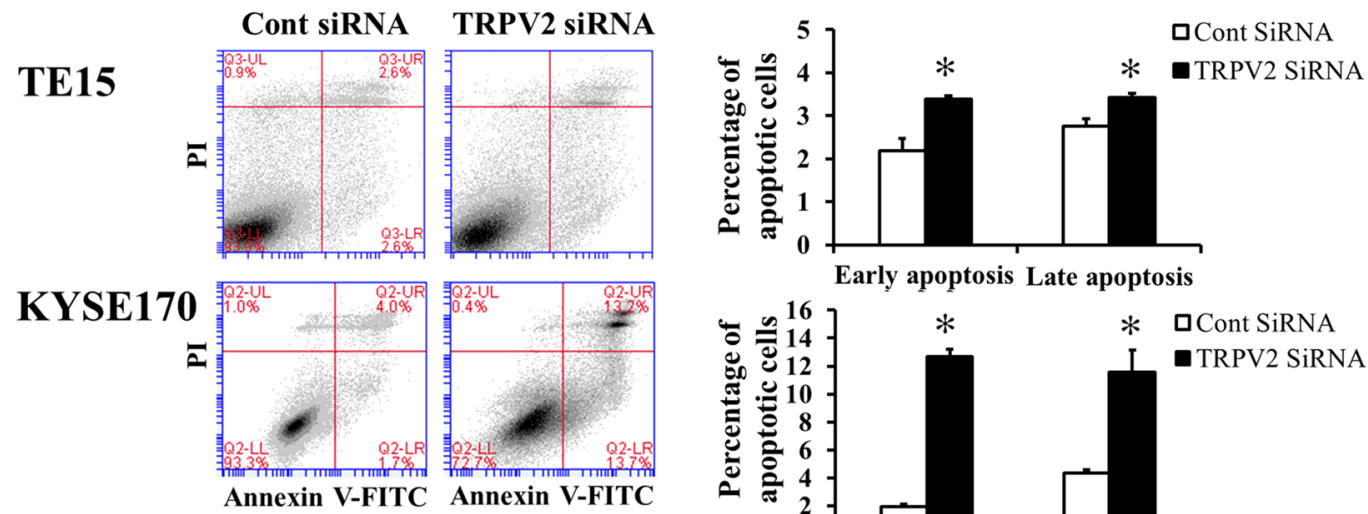

B
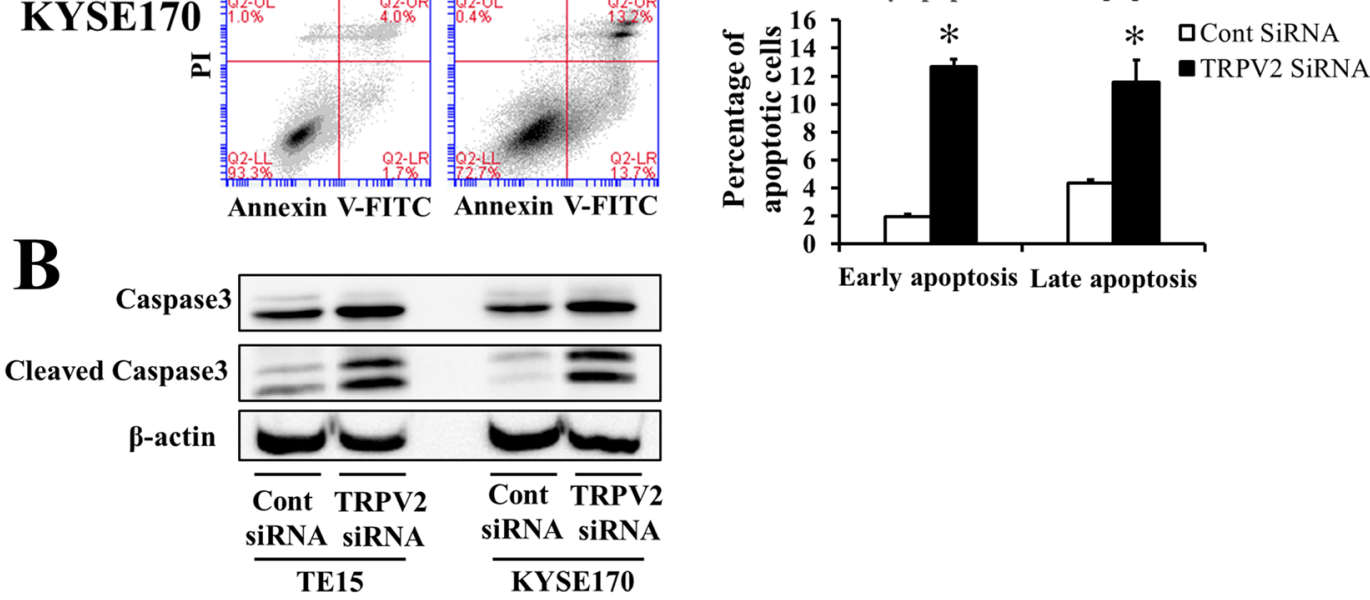

C
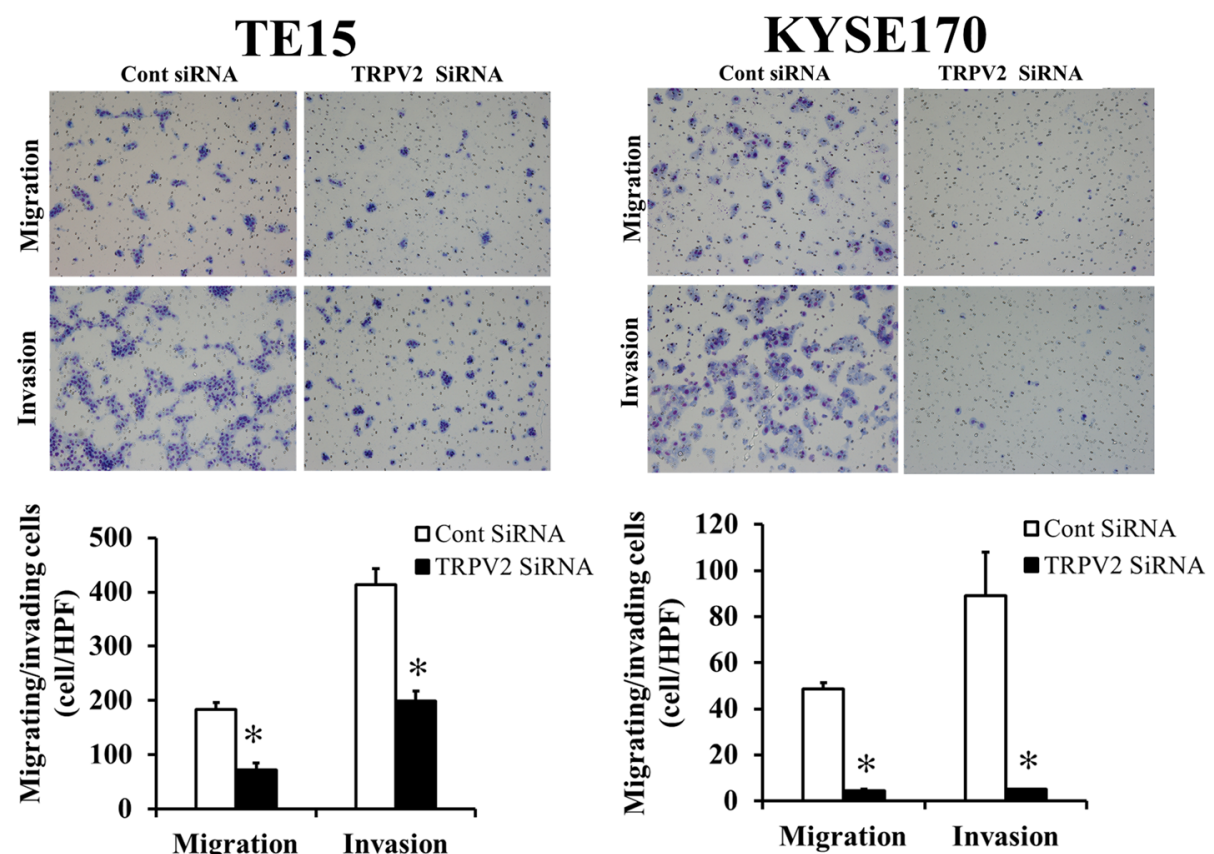

Figure 2. TRPV2 controls the survival, migration, and invasion of GC cells. (A) The down-regulation of TRPV2 induced early and late apoptosis in TE15 and KYSE170 cells. Apoptosis was assessed by flow cytometry using PI/annexin V double staining. Mean \pm SEM. $n=3 .{ }^{*} p<0.05$ (significantly different from control siRNA). (B) Apoptosis marker expression, caspase 3 and cleaved caspase 3, of ESCC cell lines transfected with control and TRPV2 siRNA was evaluated by western blotting. The western blotting figure indicated the clopped image of the bands on same gels (full-length image: Supplementary Fig. 5). The findings revealed that the expression of cleaved caspase 3, an apoptosis marker, was increased in TRPV2-depleted TE15 and KYSE170 cells. (C) The down-regulation of TRPV2 inhibited the migration and invasion of TE15 and KYSE170 cells. Cell migration and invasion were examined using the Boyden chamber assay. Mean \pm SEM. $\mathrm{n}=3 .{ }^{*} p<0.05$ (significantly different from control siRNA).

Valencia, CA). mRNA expression levels were measured by quantitative real -time PCR (7300 Real-Time PCR System; Applied Biosystems, Foster City, CA) using TaqMan Gene Expression Assays (Applied Biosystems) in accordance with the manufacturer's instructions. Expression levels were measured for the following genes: 


\begin{tabular}{|l|l|l|l|l|}
\hline Diseases or Functions & $\boldsymbol{p}$ value $(-\mathbf{l o g})$ & Predicted activation & Activation Z-score & Number of molecules \\
\hline Cell movement & 16.42 & Decreased & -5.912 & 635 \\
\hline Invasion of cells & 15.68 & Decreased & -4.633 & 293 \\
\hline Migration of cells & 14.82 & Decreased & -5.342 & 564 \\
\hline Angiogenesis & 12.45 & Decreased & -3.227 & 295 \\
\hline Migration of tumor cell lines & 11.93 & Decreased & -5.019 & 241 \\
\hline Cell movement of tumor cell lines & 11.63 & Decreased & -5.602 & 286 \\
\hline Abnormality in limbs & 11.50 & & -1.888 & 112 \\
\hline Development of vasculature & 11.17 & Decreased & -3.227 & 322 \\
\hline Vasculogenesis & 10.64 & Decreased & -3.18 & 243 \\
\hline Benign lesion & 10.47 & & 0.922 & 320 \\
\hline Invasion of tumor cell lines & 10.08 & Decreased & -4.777 & 216 \\
\hline Quantity of cells & 10.06 & Decreased & -5.065 & 498 \\
\hline Cell proliferation of tumor cell lines & 9.95 & Decreased & -4.911 & 452 \\
\hline Abnormal morphology in body cavities & 9.82 & & & 330 \\
\hline Necrosis of epithelial tissue & 9.66 & & -0.081 & 189 \\
\hline Apoptosis & 9.61 & & 1.486 & 691 \\
\hline Development of the body trunk & 9.51 & & -1.3 & 326 \\
\hline Development of epithelial tissue & 9.45 & & -1.765 & 167 \\
\hline Growth of lesions & 9.20 & Decreased & -3.186 & 250 \\
\hline Benign neoplasia & 9.20 & & 0.404 & 286 \\
\hline
\end{tabular}

Table 1. Gene ontology using IPA software.

TRPV2 (Hs00901640_m1), WNT10A(Hs00228741_m1), TGFß2(Hs00234244_m1), TGF32R(Hs00559661_m1), and Gli1 (Hs00171790_m1), Snail (Hs00195591_m1), Zeb2 (Hs00207691_m1), CDH2 (Hs00983056_m1), CD44 (Hs00153304_m1), SOX2 (Hs01053049_m1) (Applied Biosystems). Gene expression was normalized to the housekeeping gene $\beta$-actin (Hs01060665 g1; Applied Biosystems). Assays were performed in triplicate.

Analysis of cell migration and invasion. The migration assay was conducted using a Cell Culture Insert with a pore size of $8 \mu \mathrm{m}$ (BD Biosciences, Bedford, MA, USA) by our previously reported protocols ${ }^{20}$. Biocoat Matrigel (BD Biosciences) was used to evaluate cell invasion potential. Cells (TE15: $3.25 \times 10^{5}$ cells per well, KYSE170: $0.6 \times 10^{5}$ cells per well) were seeded in the upper chamber in serum-free medium $24 \mathrm{~h}$ after siRNA transfection. The lower chamber contained medium with $10 \%$ FBS. The chambers were incubated at $37^{\circ} \mathrm{C}$ for $48 \mathrm{~h}$ in $5 \% \mathrm{CO}_{2}$, and non-migrating or non-invading cells were then removed from the upper side of the membrane by scrubbing with cotton swabs. Migrating or invading cells were fixed on the membrane and stained with Diff-Quick staining reagents (Sysmex, Kobe, Japan). The migrating or invading cells on the lower side of the membrane were counted in four independent fields of view at $\times 100$ magnification for each insert. Each assay was performed in triplicate.

Microarray sample preparation and hybridization. Microarray analysis was conducted using our previously reported protocols ${ }^{20}$. Briefly, total RNA was extracted using a RNeasy kit (Qiagen). RNA quality was monitored with an Agilent 2100 Bioanalyzer (Agilent Technologies, Santa Clara, CA). Cyanine-3 (Cy3)-labeled cRNA was prepared from $0.1 \mu \mathrm{g}$ of total RNA using a Low Input Quick Amp Labeling Kit (Agilent) in accordance with the manufacturer's instructions. Samples were purified using RNeasy columns (Qiagen). A total of $0.60 \mu \mathrm{g}$ of Cy3-labeled RNA was fragmented and hybridized to an Agilent SurePrintG3 Human Gene Expression $8 \times 60 \mathrm{~K}$ ver3.0 Microarray for $17 \mathrm{~h}$. Slides were washed and scanned immediately using an Agilent DNA Microarray Scanner (G2565CA) in the one color scan setting for $8 \times 60 \mathrm{~K}$ array slides.

Processing of microarray data. Processing of microarray data was conducted using our previously reported protocols ${ }^{20}$. Scanned images were evaluated with Feature Extraction Software 10.10 (Agilent) using default parameters to acquire background-subtracted and spatially detrended processed signal intensities ${ }^{20}$. Signal transduction networks were subsequently analyzed using an Ingenuity Pathway Analysis (IPA) (Ingenuity Systems, Qiagen, Redwood City, CA) ${ }^{20}$.

Patients and primary tissue samples. ESCC human samples were obtained from 62 patients with histologically confirmed primary ESCC undergoing esophagectomy at Kyoto Prefectural University of Medicine between 1999 and 2009. All of them were embedded in paraffin after $12 \mathrm{~h}$ of formalin fixation just after the surgery. We excluded patients with non-curative resected tumors, synchronous or metachronous cancers (in addition to ESCC), preoperative chemotherapy, or radiation therapy. All eligible patients provided written informed consent. The data of relevant clinicopathological and survival were obtained from the hospital database. Staging was principally diagnosed based on the International Union Against Cancer/Tumor Node metastasis Classification of Malignant Tumors ( $7^{\text {th }}$ edition $)^{21}$. 


\begin{tabular}{|l|l|l|l|}
\hline Ingenuity Canonical Pathways & $\boldsymbol{p}$ value $(-\mathbf{l o g})$ & Predicted Activation & Activated z-score \\
\hline Human Embryonic Stem Cell Pluripotency & 5.43 & & \\
\hline Regulation of the Epithelial-Mesenchymal Transition Pathway & 4.29 & & \\
\hline Role of Osteoblasts, Osteoclasts and Chondrocytes in Rheumatoid Arthritis & 4.26 & & \\
\hline Role of Tissue Factor in Cancer & 4.03 & & \\
\hline Axonal Guidance Signaling & 3.96 & & \\
\hline Basal Cell Carcinoma Signaling & 3.77 & Decreased & -1.886 \\
\hline Role of JAK1 and JAK3 in $\gamma$ Cytokine Signaling & 3.77 & \\
\hline UVA-induced MAPK Signaling & 3.45 & Decreased & -2.065 \\
\hline Role of NANOG in Mammalian Embryonic Stem Cell Pluripotency & 3.32 & Decreased & -2 \\
\hline Glioblastoma Multiforme Signaling & 3.21 & Decreased & -2.92 \\
\hline Wnt/3-catenin Signaling & 3.04 & Decreased & -1.441 \\
\hline Molecular Mechanisms of Cancer & 3.01 & & \\
\hline Ovarian Cancer Signaling & 2.85 & Decreased & -2.183 \\
\hline IL-4 Signaling & 2.74 & & \\
\hline ERK5 Signaling & 2.55 & Decreased & -2.828 \\
\hline Colorectal Cancer Metastasis Signaling & 2.52 & Decreased & -3.221 \\
\hline Growth Hormone Signaling & 2.46 & Decreased & -1.342 \\
\hline Acute Myeloid Leukemia Signaling & 2.32 & Decreased & -1.706 \\
\hline p53 Signaling & 2.32 & Increased & 0.209 \\
\hline Phospholipases & 2.28 & & \\
\hline
\end{tabular}

Table 2. Pathway analysis using IPA software.

Immunohistochemistry. TRPV2 protein expressions in human ESCC tissues were evaluated by the same immunohistochemical staining protocol as our previous study ${ }^{20}$. Paraffin sections of tumor tissues (thickness of $4 \mu \mathrm{m}$ ) were subjected to immunohistochemical staining using the avidin-biotin-peroxidase method ${ }^{20}$. Briefly, paraffin sections were dewaxed with xylene and hydrated with a graded series of alcohol ${ }^{20}$. Endogenous peroxidases were quenched by incubating the sections for $30 \mathrm{~min}$ in $0.3 \% \mathrm{H}_{2} \mathrm{O}_{2}{ }^{20}$. The Avidin/Biotin Blocking Kit (Vector Laboratories, Burlingame, CA) was used for the blocking of endogenous biotin, biotin receptors, and avidin-binding sites ${ }^{20}$. Sections were then treated with a protein blocker and incubated at $4{ }^{\circ} \mathrm{C}$ overnight with the primary antibody ${ }^{20}$. The avidin-biotin-peroxidase complex (Vectastain ABC Elite kit; Vector Laboratories, Burlingame, CA) was visualized using diaminobenzidine tetrahydrochloride ${ }^{20}$. Sections were counterstained with hematoxylin ${ }^{20}$. These sections were then dehydrated through a graded series of alcohols, cleared in xylene, and mounted $^{20}$. Immunohistochemical samples stained with TRPV2 were graded based on staining intensities, strong or weak expression, and proportions ${ }^{20}$.

Statistical analysis. Statistical analysis was performed using same method of our previous study ${ }^{20}$. Fisher's exact test was used to evaluate differences between proportions, and the Student's $t$-test was employed to evaluate continuous variables ${ }^{20}$. Survival curves were constructed using the Kaplan-Meier method, and differences in survival were examined using the Log-rank test ${ }^{20}$. A multivariate analysis of the factors influencing survival was performed using Cox's proportional hazard model $^{20}$. Differences were considered to be significant when the relevant $p$ value was $<0.05^{20}$. These analyses were performed using JMP statistical software (version 12, SAS Institute Inc., Cary, NC) ${ }^{20}$.

\section{Results}

TRPV2 protein and mRNA expression in ESCC cell lines. Quantitative RT-PCR and Western blotting were performed to evaluate TRPV2 expression in the human ESCC cell lines, TE5, TE8, TE9, TE15, KYSE70, LYSE150, and KYSE170 (Fig. 1A,B). TRPV2 mRNA was strongly expressed in TE15, and more strongly expressed in KYSE170 than in the other ESCC cell lines. Meanwhile, similar intensity of TRPV2 protein expression was observed in TE5, TE9, TE15, KYSE70 and KYSE170.

siRNA knockdown of TRPV2 suppressed the proliferation of ESCC cells. We conducted knockdown experiments using siRNA in the TRPV2-overexpressing ESCC cell lines, TE15 and KYSE170, and evaluated the effects of TRPV2 depletion on cell proliferation. TRPV2 siRNA effectively decreased TRPV2 mRNA and protein expression in TE15 and KYSE170 (Fig. 1C). Cell proliferation was significantly less in TRPV2-depleted cells than in control-siRNA-treated cells (Fig. 1D).

TRPV2 depletion inhibited cell cycle progression and induced apoptosis in ESCC cells. A cell cycle analysis was performed to investigate the mechanisms underlying suppressed proliferation induced by TRPV2 depletion in ESCC cells (Fig. 1E). The transfection of TRPV2 siRNA partially inhibited cell cycle progression from the G1 to S phase in TE15 and KYSE170. Moreover, the cell population of sub-G1 increased in TPRV2-depleted cells, suggesting that TRPV2 depletion suppressed the proliferation of ESCC cells by inhibiting cell cycle progression or inducing apoptosis. In order to validate the induction of apoptosis by TRPV2 

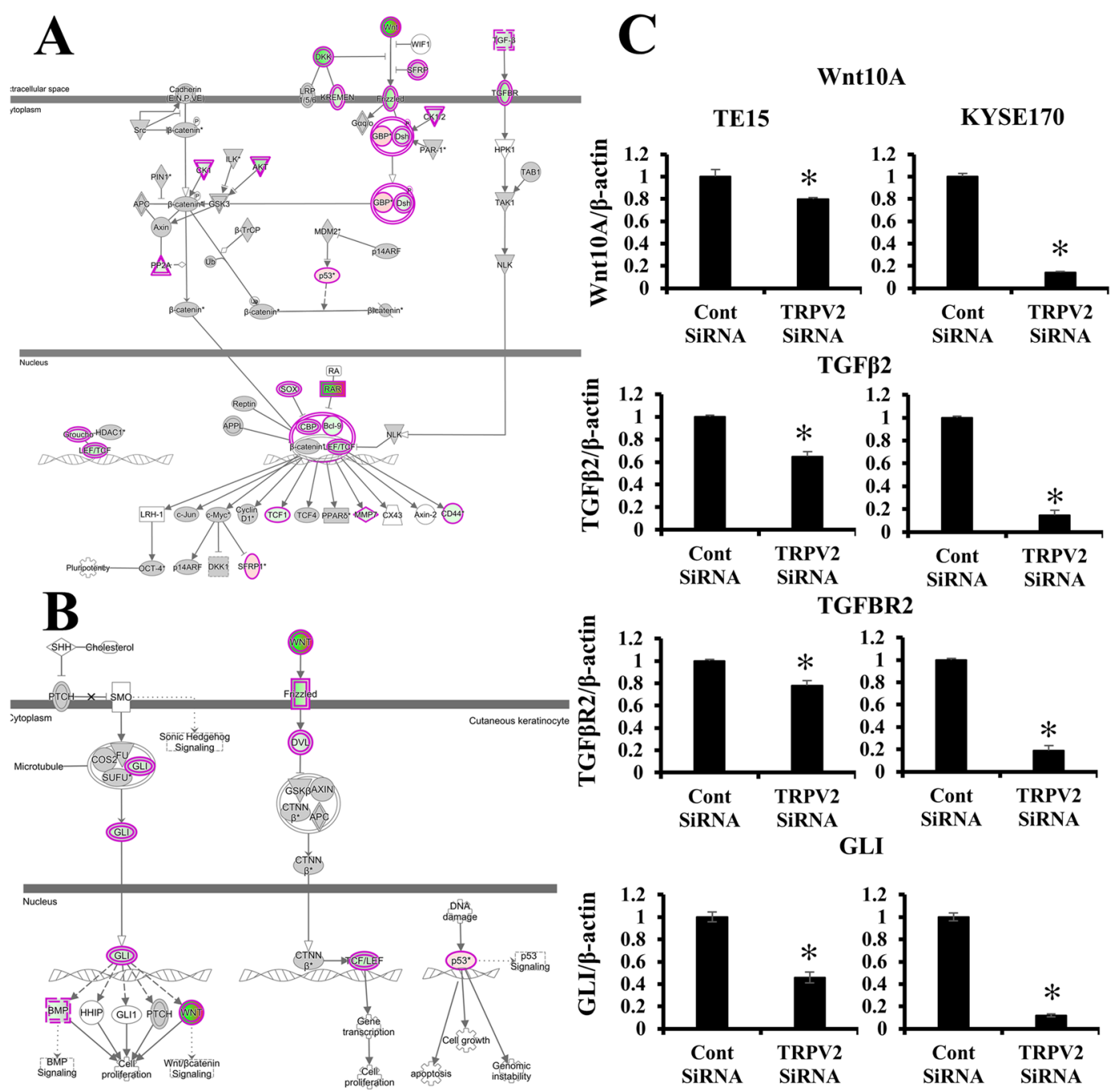

Figure 3. Signal pathways regulated by TRPV2 in ESCC cells. (A) (A) The signaling map, which was generated using an Ingenuity Pathway Analysis (IPA) software (Ingenuity Systems, Qiagen, Redwood City, CA), of

"WNT/ $\beta$-Catenin", the top-ranking canonical pathway related to TRPV2 depletion according to an IPA analysis. Red and green indicate genes with expression levels that were higher or lower, respectively, than reference RNA levels. (B) The signaling map, which was generated by IPA software, of "Basal cell carcinoma signaling" (C)

Verification of gene expression by real-time quantitative RT-PCR. The expression levels of four selected "WNT/ TGF- $\beta$ Catenin" and "Basal cell carcinoma signaling"-related genes (WNT10A, TGF- $\beta 2$, TGF- $\beta 2$ R, and GLI) in TRPV2-depleted TE15 and KYSE170 were compared to those in control siRNA-transfected cells using real-time quantitative RT-PCR. Mean \pm SEM. $\mathrm{n}=3 .{ }^{*} p<0.05$ (significantly different from control siRNA).

depletion, an apoptosis assay and Western blotting of the apoptosis markers, caspase 3 and cleaved caspase 3 were conducted. The results obtained revealed that the down-regulation of TRPV2 induced early apoptosis (annexin V-positive/PI negative) and late apoptosis (annexin V-positive/PI positive) in TE15 and KYSE170 72 hours after the siRNA knockdown (Fig. 2A). Western blotting showed that the expression of cleaved caspase 3 increased in TRPV2-depleted ESCC cells (Fig. 2B).

TRPV2 controlled cell migration and invasion in ESCC cells. The effects of the down-regulation of TRPV2 on cell migration and invasion in ESCC cells were analyzed, and the results obtained revealed that the TRPV2 siRNA knockdown inhibited cell migration and invasion (Fig. 2C). These results indicated that TRPV2 plays an important role in the cancer cell migration and invasion of ESCC.

Gene expression profiles of TRPV2-depleted ESCC cells. The gene expression profile of TRPV2-depleted KYSE170 cells was analyzed using microarrays and a bioinformatic study. Microarray results showed that the expression of 3697 genes displayed a fold change of $>2.0$ in KYSE170 cells upon the depletion of TRPV2. The number of up-regulated genes was 1372, while that of down-regulated genes was 2325. A list of 20 genes with expression levels that were the most strongly up- or down-regulated in TRPV2-depleted KYSE170 cells was shown in Supplementary Tables 1 and 2. The $\mathrm{Ca}^{2+}$-related genes, MICU1 and CACNB2, were strongly 

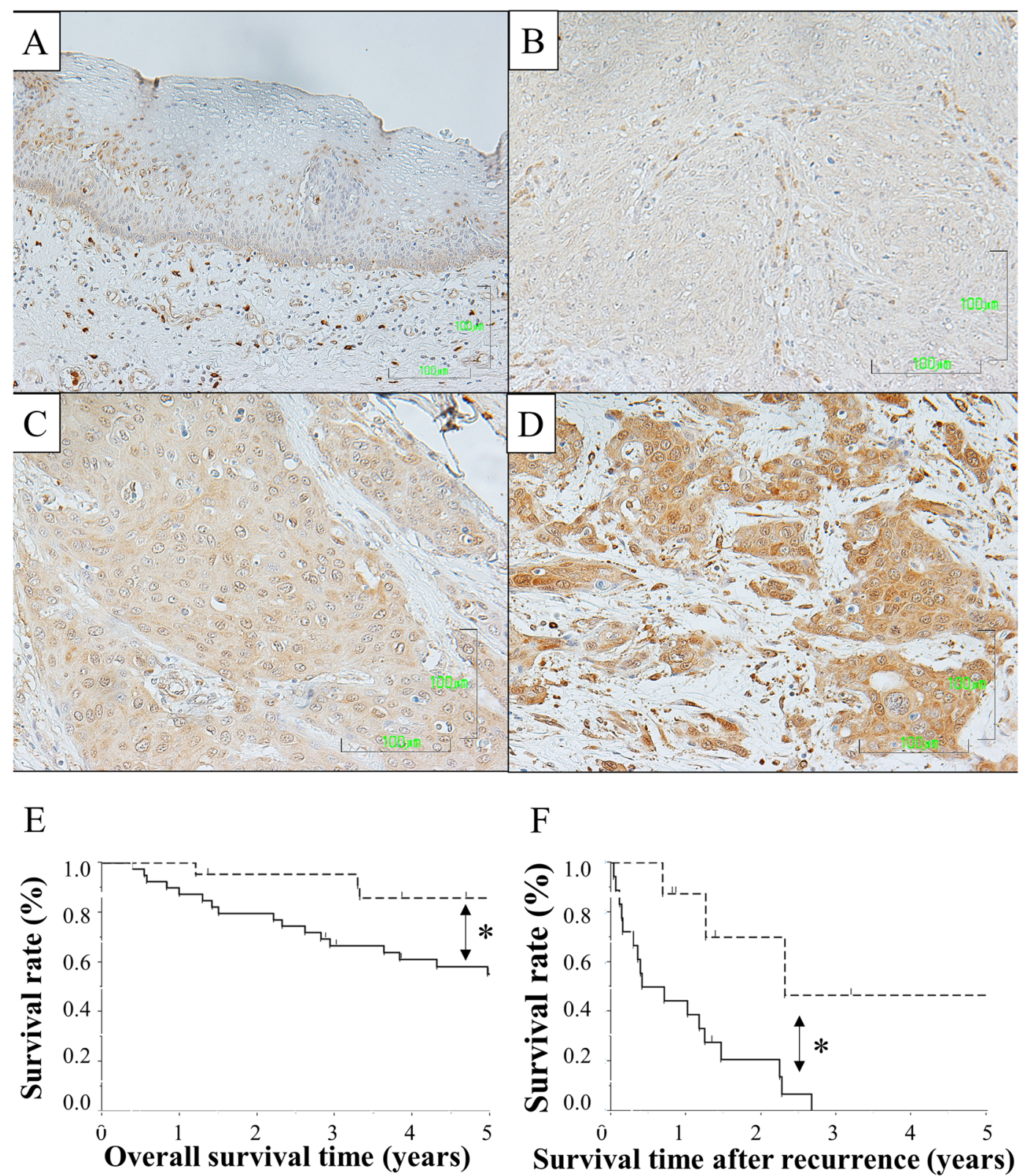

$\mathrm{F}$

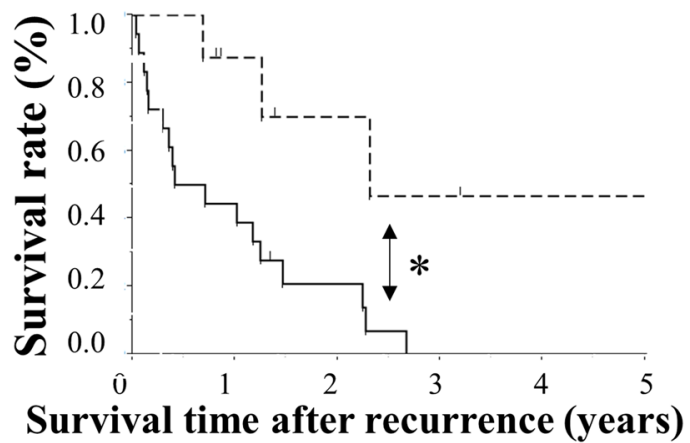

Figure 4. TRPV2 protein expression in human ESCCs. (A) Immunohistochemical staining of primary ESCC samples showed that nuclear TRPV2 expression was detected in the middle layer of the non-cancerous stratified squamous epithelium. Magnification: $\times 400$. Bar, $100 \mu \mathrm{m}$. (B) Immunohistochemical staining of primary human ESCC samples without TRPV2 expression. Magnification: $\times 400$. Bar, $100 \mu \mathrm{m}$. (C) Immunohistochemical staining of primary human ESCC samples with the weak cytoplasmic expression of TRPV2. Magnification: $\times 400$. Bar, $100 \mu \mathrm{m}$. (D) Immunohistochemical staining of primary human ESCC samples with the strong cytoplasmic expression of TRPV2. Magnification: $\times 400$. Bar, $100 \mu \mathrm{m}$. (E) Survival curve of patients after curative resection for ESCC according to the expression of TRPV2. All patients were classified into two groups according to the proportion of strong TRPV2 expression in ESCC tumors: low group: $<20 \%$, high group: $\geq 20 \%$ : the low group $(n=22)$ and high group $(n=40)$ in the tumor. ${ }^{*} p<0.05$ : Log-rank test. (F) Survival curve of ESCC patients after post-operative recurrence $(n=26)$ according to the expression of TRPV2. All patients were classified into two groups according to the proportion of strong TRPV2 expression in ESCC tumors: low group: $<20 \%$, high group: $\geq 20 \%$ : the low group $(n=8)$ and high group $(n=18)$ in the tumor. $* p<0.05:$ Logrank test.

down-regulated in TRPV2-depleted KYSE170 cells. Furthermore, a gene ontology analysis (IPA) showed that the function of "the invasion of cells", "migration of cells", "migration of tumor cell lines", "invasion of tumor cell lines", "cell proliferation of tumor cell lines", "apoptosis", and "cell proliferation of tumor cell lines" were down-regulated (activation z-score, -3.156 to -5.912 ) (Table 1). These results were consistent with those of the cancer function, proliferation, cell cycle, apoptosis, and migration/invasion assays. 


\begin{tabular}{|c|c|c|c|c|}
\hline & & $\begin{array}{l}\text { Low group } \\
(\mathrm{n}=22)\end{array}$ & $\begin{array}{l}\text { High group } \\
(\mathrm{n}=40)\end{array}$ & $p$ value \\
\hline \multirow[t]{2}{*}{ Sex } & Male & 18 & 36 & 0.367 \\
\hline & Female & 4 & 4 & \\
\hline \multirow[t]{2}{*}{ Age } & $<65$ & 14 & 21 & 0.221 \\
\hline & $\geq 65$ & 8 & 19 & \\
\hline \multirow[t]{2}{*}{ Histology type } & Well/Moderate & 18 & 27 & 0.217 \\
\hline & Poor & 4 & 13 & \\
\hline \multirow[t]{2}{*}{ Location } & Ce-Ut & 4 & 5 & 0.432 \\
\hline & Mt-Lt & 18 & 35 & \\
\hline \multirow[t]{2}{*}{ Tumor size $(\mathrm{mm})$} & $<50$ & 17 & 25 & 0.082 \\
\hline & $\geq 50$ & 3 & 14 & \\
\hline \multirow[t]{2}{*}{ Lymphatic invasion } & Negative & 10 & 19 & 0.877 \\
\hline & Positive & 12 & 21 & \\
\hline \multirow[t]{2}{*}{ Venous invasion } & Negative & 15 & 20 & 0.164 \\
\hline & Positive & 7 & 20 & \\
\hline \multirow[t]{2}{*}{$\mathrm{pT}$} & pT1 & 13 & 18 & 0.287 \\
\hline & pT2-4 & 9 & 22 & \\
\hline \multirow[t]{2}{*}{$\mathrm{pN}$} & $\mathrm{pN} 0$ & 11 & 19 & 0.851 \\
\hline & $\mathrm{pN} 1-3$ & 11 & 21 & \\
\hline \multirow[t]{3}{*}{ Recurrence } & All & 8 & 18 & 0.509 \\
\hline & Local recurrence & 4 & 6 & 0.744 \\
\hline & Distant recurrence & 4 & 12 & 0.150 \\
\hline Initial treatment for recurrence & Radical treatment & 4 & 4 & \\
\hline
\end{tabular}

Table 3. Correlations between clinicopathological features and TRPV2 expression. Ce: cervical esophagus, Ut: upper thoracic esophagus, Mt: middle thoracic esophagus, Lt: lower thoracic esophagus. pT: pathological tumor invasion depth, pN: pathological lymph node metastasis, Radical treatment: surgery or chemoradiotherapy.

\begin{tabular}{|c|c|c|c|c|c|c|c|}
\hline & & \multirow[b]{2}{*}{$\mathbf{n}$} & \multicolumn{2}{|c|}{ Univariable } & \multicolumn{2}{|c|}{ Multivariable } & \multirow[b]{2}{*}{$p$ value } \\
\hline & & & 5-year OS & p value & HR & $95 \% \mathrm{CI}$ & \\
\hline \multirow[t]{2}{*}{ Sex } & Male & 54 & $62.9 \%$ & 0.199 & & & \\
\hline & Female & 8 & $87.5 \%$ & & & & \\
\hline \multirow[t]{2}{*}{ Age } & $<65$ & 33 & $65.9 \%$ & 0.939 & & & \\
\hline & $\geq 65$ & 29 & $66.6 \%$ & & & & \\
\hline \multirow[t]{2}{*}{ Histology type } & Well/Moderate & 45 & $71.5 \%$ & 0.156 & & & \\
\hline & Poor & 17 & $52.9 \%$ & & & & \\
\hline \multirow[t]{2}{*}{ Lymphatic invasion } & Negative & 29 & $70.1 \%$ & 0.522 & & & \\
\hline & Positive & 33 & $62.3 \%$ & & & & \\
\hline \multirow[t]{2}{*}{ Venous invasion } & Negative & 35 & $78.9 \%$ & 0.012 & 2.437 & $0.983-6.576$ & 0.054 \\
\hline & Positive & 27 & $49.3 \%$ & & & & \\
\hline \multirow[t]{2}{*}{$\mathrm{pT}$} & pT1 & 31 & $73.1 \%$ & 0.165 & & & \\
\hline & pT2-4 & 31 & $59.4 \%$ & & & & \\
\hline \multirow[t]{2}{*}{$\mathrm{pN}$} & pNo & 30 & $79.7 \%$ & 0.041 & 2.294 & $0.915-6.511$ & 0.077 \\
\hline & $\mathrm{pN} 1-3$ & 32 & $53.6 \%$ & & & & \\
\hline \multirow[t]{2}{*}{ TRPV2 expression } & Low group & 22 & $85.2 \%$ & 0.020 & 3.153 & $1.041-13.638$ & 0.041 \\
\hline & High group & 40 & $59.5 \%$ & & & & \\
\hline
\end{tabular}

Table 4. Prognostic factors of esophageal squamous cell carcinoma according to univariate and multivariate analyses. pT: pathological tumor invasion depth, $\mathrm{pN}$ : pathological lymph node metastasis.

Pathway analysis and molecular mechanisms regulated by TRPV2 in ESCC cells. We examined the signal transduction networks induced by the TRPV2 knockdown using IPA. The results obtained are shown in Table 2. "WNT/ $\beta$-Catenin signaling", "basal cell Carcinoma signaling", and "regulation of the Epithelial-Mesenchymal Transition Pathway" were the top-ranking canonical pathways related to TRPV2 depletion, while "basal cell carcinoma signaling" and "WNT/ $\beta$-Catenin signaling" were predicted to be down-regulated pathways (activation $z$-score: -1.886 and -1.441 ) (Fig. 3A,B and Supplementary Fig. 1A). Moreover, the pathways associated with stemness, "Human Embryonic Stem Cell Pluripotency", "Role of NANOG in Mammalian Embryonic Stem Cell Pluripotency", and "Wnt//-catenin Signaling", were the top ranked in the canonical pathway. 
In order to confirm the results of the microarray analysis, the expression of several genes included in "WNT/ $\beta$-Catenin signaling", "basal cell carcinoma signaling", "regulation of the Epithelial-Mesenchymal Transition Pathway", and the relationship between stem cell-related genes and TRPV2 expression were validated in more detail using quantitative RT-PCR. WNT10A, TGF32, TGFßR2, GLI, Snai1, Zeb2, CDH2, CD44, and SOX2 mRNA expression levels were lower in TRPV2-depleted TE5 or KYSE170 than in control siRNA-transfected cells (Fig. 3C, Supplementary Figs 1B and 2). These results suggesting that TRPV2 regulated cancer cell function via "WNT/ $\beta$-Catenin signaling", "basal cell Carcinoma signaling", "regulation of the Epithelial-Mesenchymal Transition Pathway" and the expression of stem cell-related genes.

TRPV2 protein expression in human ESCC samples. In order to evaluate the significance of TRPV2 protein expression in ESCC samples, we performed immunohistochemical staining on 62 tumor samples of Human ESCC using the TRPV2 antibody. Nuclear TRPV2 expression was detected in the middle layer of the non-cancerous stratified squamous epithelium. (Fig. 4A). Although TRPV2 cytoplasmic expression in carcinoma cells varied widely between each ESCC sample, we initially evaluated TRPV2 signal intensities in cancer cells as no, weak, and strong expression (Fig. 4B-D); the proportion of each intensity in tumors was subsequently measured. ESCC samples in which the proportion of strong intensity was $20 \%$ or larger were defined as high expression $(n=40)$, and the others as low expression $(n=22)$. The relationships between TRPV2 protein expression and patient backgrounds and various clinicopathological parameters were analyzed (Table 3 ). Tumor sizes were slightly larger in the high group than in the low group. No significant difference was observed in recurrence rate; however, distant recurrence was tended to occur more frequently in TRPV2 high group. Furthermore, the number of cases who underwent radical treatment for recurrence, such as chemoradiotherapy or surgery, was tended to be larger in TRPV2 low group (50.0\% vs $38.8 \%)$.

The relationship between the expression of TRPV2 and prognosis of ESCC patients was investigated. The 5-year overall survival rate after surgery was significantly worse in the high group than in the low group (59.5\% vs $85.2 \%$, $p=0.020$ ) (Fig. 4E). Moreover, the 5-year survival of ESCC patients after post-operative recurrence in the high group was significantly worse than in the low group ( $46.6 \%$ vs $0.0 \%, p=0.006$ ) (Fig. $4 \mathrm{~F}$ ). A univariate analysis of survival (5-year overall survival) showed that pathological venous invasion, pathological lymph node metastasis, and the strong expression of TRPV2 were significant. A multivariate analysis with these 3 factors (Cox's proportional hazard model) revealed that the strong expression of TPRV2 was an independent poor prognostic factor (Table 4).

\section{Discussion}

A role for TRPV2 in cellular development or morphology was recently reported. Kojima et al. showed that TRPV2 was associated with cell cycle progression via the regulation of its translocation induced by Insulin-Like Growth Factor $1^{22}$. TRPV2 has been shown to play a role in cellular migration through the regulation of intracellular $\mathrm{Ca}^{2+}$ concentrations ${ }^{11}$. In the field of oncology, many researchers reported that TRPV2 similarly regulated cell death in cancer cells or cancer migration/invasion ${ }^{13,15,16,18,23}$. They showed that the regulation of $\mathrm{Ca}^{2+}$ signaling by TRPV2 may affect these cancer functions. $\mathrm{Ca}^{2+}$ is an essential element for the survival and function of cells. Amplifications in the magnitude and duration of intracellular $\mathrm{Ca}^{2}$ changes may mean the difference between cellular migration and cell death. In malignant cells, calcium signaling plays important roles in proliferation, apoptosis, tumor stromal interaction, metastasis, and drug resistance ${ }^{24,25}$. In the present study, TRPV2 expression was firstly evaluated, and TRPV2 knockdown experiment was subsequently performed. Although TRPV2 expression in ESCC cell lines was observed, the discrepancy existed between the protein and mRNA expression. Zhang et al. described that the intensity of protein expression was not consistent with mRNA expression in over two-third of molecules which expressed in human colorectal cancer specimens ${ }^{26}$. TRPV2 may be one of the molecules with the inconsistency between gene and protein expression. Knockdown experiments demonstrated that TRPV2 depletion suppressed tumor proliferation, cell cycle progression, and migration/invasion, and also induced apoptosis in ESCC cells (Figs 1 and 2). Moreover, the gene ontology analysis revealed that cancer functions, such as cell invasion, angiogenesis, cell migration, cell proliferation, and apoptosis, were down-regulated in TRPV2-depleted ESCC cells (Table 1). These results were consistent with the previously reported antitumor effects induced by the regulation of $\mathrm{Ca}^{2+}$ signaling. Therefore, it is plausible that TRPV2 regulates cancer biology via calcium signaling in ESCC.

Furthermore, we performed a pathway analysis to clarify the role of TRPV2 in the cancer signaling of ESCC, and revealed that the depletion of TRPV2 down-regulated "basal cell carcinoma signaling". "Basal cell carcinoma signaling" is a pathway related to proliferation or apoptosis in basal cell carcinoma, in which cross talk between the hedgehog pathway and Wnt/ $\beta$-Catenin signaling activates several cancer functions ${ }^{27,28}$. The involvement of the hedgehog pathway in ESCC was previously reported in our laboratory ${ }^{29}$. The present results indicated that TRPV2 regulated malignant potentials via cross talk between the hedgehog pathway and Wnt/ $\beta$-catenin signaling; furthermore, $\mathrm{Ca}^{2+}$ may act as a second messenger between TRPV2 expression and these pathways. Previous studies revealed that intracellular $\mathrm{Ca}^{2+}$ plays an important role in the WNT pathway (WNT/calcium pathway) ${ }^{30,31}$. In this pathway, intracellular $\mathrm{Ca}^{2+}$ act as a second messenger, resulting in the control of cancer-related gene expression. These results and previous findings suggested that TRPV2 controls WNT/ $\beta$ catenin signaling and basal cell carcinoma signaling (cross talk between the hedgehog and WNT pathways) via the regulation of $\mathrm{Ca}^{2+}$ signals, such as WNT/calcium signaling.

TRPV2 depletion also down-regulated "Wnt/ $\beta$-catenin signaling" in the pathway analysis, which regulated pluripotency via the translocation of $\beta$-catenin into the nucleus. The relationship between this pathway and cancer stem cells has already been reported ${ }^{32,33}$. In the microarray data obtained in the present study, TRPV2 depletion down-regulated the expression of the stem cell markers SOX2 and CD44. Furthermore, the top-ranked pathway contained the stemness-related signals "Human Embryonic Stem Cell Pluripotency" and "Role of NANOG in Mammalian Embryonic Stem Cell Pluripotency". The validation of gene alterations using RT-PCR 
revealed that CD44 and SOX2 were down-regulated in TRPV2-depleted ESCC cells. We previously reported the overexpression of TRPV2 in cancer stem cells derived from ESCC cell lines, and the present results from the pathway analysis were consistent with these findings. Therefore, TRPV2 may also maintain the stemness of ESCC stem cells via these pathways.

TRPV2 depletion down-regulated the gene expression of TGF $\beta$ and TGF $\beta$ R in "Wnt $/ \beta$-catenin signaling" in contrast to our exceptions. These results may be explained by the TGF $\beta$ paradox. TGF $\beta$ plays contrasting roles in tumors, acting as a tumor suppressive gene during the first stages of carcinogenesis and as a tumor promoter during the advanced stages of progression ${ }^{34,35}$. Therefore, the paradoxical results of TGF $\beta$ and TGF $\beta$ R were consistent with our hypothesis.

Moreover, the pathway analysis revealed that the TRPV2 knockdown altered the expression of many genes associated with epithelial-mesenchymal transition (Table 2, rank 2). The validation of gene alterations using RT-PCR revealed that TGF $\beta$, WNT, and SNAIL were down-regulated in TRPV2-depleted ESCC cells. These results suggested that TRPV2 controls the migration/invasion ability of ESCC via epithelial-mesenchymal transition.

Finally, we performed immunohistochemical analysis in order to evaluate clinical significance of TRPV2 expression in ESCC samples. The results revealed that TRPV2 expression was significantly associated with overall survival; however, significant correlations between clinicopathological features and TRPV2 expression were not observed. To clarify the reason of bad prognosis in TRPV2 high group, recurrent patterns and survival after recurrence were analyzed, revealing that distant recurrence were more frequently observed in TRPV2 high group and the prognosis after recurrence in TRPV2 high group was significantly worse than in low group. These findings suggested that TRPV2 affected recurrence pattern or therapeutic effect of therapy for recurrence.

In summary, we herein demonstrated that TRPV2 played a role in the proliferation, cell cycle progression, survival, migration, and invasion of ESCC cells. Microarray results showed that TRPV2 markedly affected the expression of genes related to WNT/ $\beta$-catenin signaling, basal cell carcinoma signaling (cross talk between the hedgehog and $\mathrm{WNT} / \beta$-catenin pathways), and regulation of the epithelial-mesenchymal transition pathway. The results of the immunohistochemical analysis of ESCC human samples revealed that the strong expression of TRPV2 was a poor prognostic factor in patients with ESCC. Although further investigations are needed, the present results demonstrate that TRPV2 has potential as a poor prognostic biomarker and novel therapeutic target for ESCC.

\section{Data availability}

The data sets generated during and/or analysed during the current study are available from the corresponding author on reasonable request.

Received: 16 August 2019; Accepted: 14 October 2019;

Published online: 05 November 2019

\section{References}

1. Caterina, M. J, et al The capsaicin receptor: a heat-activated ion channel in the pain pathway. Nature 389(6653), 816-824, e-pub ahead of print 1997/12/31 23:16, https://doi.org/10.1038/39807 (1997).

2. Kanzaki, M., Nie, L., Shibata, H. \& Kojima, I. Activation of a calcium-permeable cation channel CD20 expressed in Balb/c 3T3 cells by insulin-like growth factor-I. The Journal of biological chemistry 272(8), 4964-4969, https://doi.org/10.1074/jbc.272.8.4964 (1997). e-pub ahead of print 1997/02/21.

3. Smith, G. D. et al. TRPV3 is a temperature-sensitive vanilloid receptor-like protein. Nature 418(6894), 186-190, e-pub ahead of print 2002/06/22, https://doi.org/10.1038/nature00894 (2002).

4. Strotmann, R., Harteneck, C., Nunnenmacher, K. \& Schultz, G., Plant TD. OTRPC4, a nonselective cation channel that confers sensitivity to extracellular osmolarity. Nature cell biology 2(10), 695-702, e-pub ahead of print 2000/10/12, https://doi. org/10.1038/35036318 (2000).

5. Liedtke, W. et al. Vanilloid receptor-related osmotically activated channel (VR-OAC), a candidate vertebrate osmoreceptor. Cell 103(3), 525-535, e-pub ahead of print 2000/11/18 (2000).

6. Caterina, M. J., Rosen, T. A., Tominaga, M., Brake, A. J. \& Julius, D. A capsaicin-receptor homologue with a high threshold for noxious heat. Nature 398(6726), 436-441, e-pub ahead of print 1999/04/14, https://doi.org/10.1038/18906 (1999).

7. Samanta, A., Hughes, T. E. T. \& Moiseenkova-Bell, V. Y. Transient Receptor Potential (TRP) Channels. Subcell Biochem 87, 141-165, https://doi.org/10.1007/978-981-10-7757-9_6(2018).

8. Mihara, H. et al. Involvement of TRPV2 activation in intestinal movement through nitric oxide production in mice. $J$ Neurosci 30(49), 16536-16544, https://doi.org/10.1523/JNEUROSCI.4426-10.2010 (2010).

9. Hisanaga, E. et al. Regulation of calcium-permeable TRPV2 channel by insulin in pancreatic beta-cells. Diabetes 58(1), 174-184, https://doi.org/10.2337/db08-0862 (2009).

10. Issa, C. M. et al. TRPV2 in the development of experimental colitis. Scand J Immunol 80(5), 307-312, https://doi.org/10.1111/ sji.12206 (2014).

11. Gambade, A. et al. Activation of TRPV2 and BKCa channels by the LL-37 enantiomers stimulates calcium entry and migration of cancer cells. Oncotarget 7(17), 23785-23800, e-pub ahead of print 2016/03/20, https://doi.org/10.18632/oncotarget.8122 (2016).

12. Oulidi, A. et al. TRPV2 mediates adrenomedullin stimulation of prostate and urothelial cancer cell adhesion, migration and invasion. PLoS One 8(5), e64885, https://doi.org/10.1371/journal.pone.0064885 (2013).

13. Liu, Q. \& Wang, X. Effect of TRPV2 cation channels on the proliferation, migration and invasion of 5637 bladder cancer cells. Exp Ther Med 6(5), 1277-1282, https://doi.org/10.3892/etm.2013.1301 (2013).

14. Zoppoli, P. et al. TRPV2 Calcium Channel Gene Expression and Outcomes in Gastric Cancer Patients: A Clinically Relevant Association. J Clin Med 8(5), https://doi.org/10.3390/jcm8050662 (2019).

15. Elbaz, M. et al. TRPV2 is a novel biomarker and therapeutic target in triple negative breast cancer. Oncotarget 9(71), 33459-33470, e-pub ahead of print 2016/05/27, https://doi.org/10.18632/oncotarget.9663 (2018).

16. Liberati, S. et al. Loss of TRPV2 Homeostatic Control of Cell Proliferation Drives Tumor Progression. Cells 3(1), 112-128, https:// doi.org/10.3390/cells3010112 (2014).

17. Shiozaki, A. et al. Esophageal cancer stem cells are suppressed by tranilast, a TRPV2 channel inhibitor. J Gastroenterol 53(2), 197-207, https://doi.org/10.1007/s00535-017-1338-x (2018). 
18. Zhou, K., Zhang, S. S., Yan, Y. \& Zhao, S. Overexpression of transient receptor potential vanilloid 2 is associated with poor prognosis in patients with esophageal squamous cell carcinoma. Med Oncol 31(7), 17, https://doi.org/10.1007/s12032-014-0017-5 (2014).

19. Ministry to Health, Labor and Welfare, Ethical Guidelines for Medical and Health Research Involving Human Subjects, https://www. mhlw.go.jp/stf/seisakunitsuite/bunya/hokabunya/kenkyujigyou/i-kenkyu/index.html.

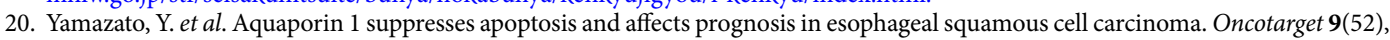
29957-29974, e-pub ahead of print 2018/07/26, https://doi.org/10.18632/oncotarget.25722 (2018).

21. Sobin, L. H. \& Compton, C. C. TNM seventh edition: what's new, what's changed: communication from the International Union Against Cancer and the American Joint Committee on Cancer. Cancer 116(22), 5336-5339, e-pub ahead of print 2010/07/29, https://doi.org/10.1002/cncr.25537 (2010).

22. Kojima, I \& Nagasawa, M. Chapter 7 TRPV2: A Calcium-Permeable Cation Channel Regulated by Insulin-Like Growth Factors, TRP Ion Channel Function in Sensory Transduction and Cellular Signaling Cascades. Boca Raton (FL): CRC Press/Taylor \& Francis. 2007.

23. Santoni, G. \& Amantini, C. The Transient Receptor Potential Vanilloid Type-2(TRPV2) Ion Channels in Neurogenesis andGliomagenesis: Cross-Talk between TranscriptionFactors and Signaling Molecules. Cancers (Basel) 11(3), https://doi. org/10.3390/cancers11030322 (2019).

24. Xu, M., Seas, A., Kiyani, M., Ji, K. S. Y. \& Bell, H. N. A temporal examination of calcium signaling in cancer- from tumorigenesis, to immune evasion, and metastasis. Cell Biosci 8, 25, https://doi.org/10.1186/s13578-018-0223-5 (2018).

25. Monteith, G. R., McAndrew, D., Faddy, H. M. \& Roberts-Thomson, S. J. Calcium and cancer: targeting $\mathrm{Ca}^{2+}$ transport. Nat Rev Cancer 7(7), 519-530, https://doi.org/10.1038/nrc2171 (2007).

26. Zhang, B. et al. Proteogenomic characterization of human colon and rectal cancer. Nature 513(7518), 382-387 (2014).

27. Mimeault, M. \& Batra, S. K. Frequent deregulations in the hedgehog signaling network and cross-talks with the epidermal growth factor receptor pathway involved in cancer progression and targeted therapies. Pharmacol Rev 62(3), 497-524, https://doi. org/10.1124/pr.109.002329 (2010)

28. Abe, Y \& Tanaka, N. Roles of the Hedgehog Signaling Pathway in Epidermal and Hair Follicle Development, Homeostasis, and Cancer. J Dev Biol 5(4), https://doi.org/10.3390/jdb5040012 (2017).

29. Shiozaki, A. et al. Expression and role of anion exchanger 1 in esophageal squamous cell carcinoma. Oncotarget 8(11), 17921-17935, e-pub ahead of print 2017/02/06, https://doi.org/10.18632/oncotarget.14900 (2017).

30. Zhan, T., Rindtorff, N. \& Boutros, M. Wnt signaling in cancer. Oncogene 36(11), 1461-1473, https://doi.org/10.1038/onc.2016.304 (2017).

31. De, A. Wnt/Ca ${ }^{2+}$ signaling pathway: a brief overview. Acta biochimica et biophysica Sinica 43(10), 745-756, e-pub ahead of print 2011/09/10, https://doi.org/10.1093/abbs/gmr079 (2011).

32. Valkenburg, K. C., Graveel, C. R., Zylstra-Diegel, C. R., Zhong, Z. \& Williams, B. O. Wnt/beta-catenin Signaling in Normal and Cancer Stem Cells. Cancers (Basel) 3(2), 2050-2079, https://doi.org/10.3390/cancers3022050 (2011).

33. de Sousa, E. M. F. \& Vermeulen, L. Wnt Signaling in Cancer Stem Cell Biology. Cancers (Basel) 8(7), https://doi.org/10.3390/ cancers8070060 (2016).

34. Neuzillet, C. et al. Targeting the TGFbeta pathway for cancer therapy. Pharmacol Ther 147, 22-31, https://doi.org/10.1016/j. pharmthera.2014.11.001 (2015).

35. Cantelli, G., Crosas-Molist, E., Georgouli, M. \& Sanz-Moreno, V. TGFBeta-induced transcription in cancer. Semin Cancer Biol 42, 60-69, https://doi.org/10.1016/j.semcancer.2016.08.009 (2017)

\section{Acknowledgements}

This work was supported by Grants-in-Aid for Scientific Research (C), 17K10602, 17K10710, 18K08628, and 18K08689, and a Grant-in-Aid for Research Activity start- up, 17H07014.

\section{Author contributions}

M.K., A.S., M.K., E.K., Y.M., E.O. designed the research and wrote the paper; Y.Y., K.K., performed cell culture, molecular biology and several experiments; T.K., K.S., T.A., H.K., S.K., T.K., H.F. and K.O. provided clinical specimens and performed clinical data analyses.

\section{Competing interests}

The authors declare no competing interests.

\section{Additional information}

Supplementary information is available for this paper at https://doi.org/10.1038/s41598-019-52227-0.

Correspondence and requests for materials should be addressed to A.S.

Reprints and permissions information is available at www.nature.com/reprints.

Publisher's note Springer Nature remains neutral with regard to jurisdictional claims in published maps and institutional affiliations.

(c) (i) Open Access This article is licensed under a Creative Commons Attribution 4.0 International (c) License, which permits use, sharing, adaptation, distribution and reproduction in any medium or format, as long as you give appropriate credit to the original author(s) and the source, provide a link to the Creative Commons license, and indicate if changes were made. The images or other third party material in this article are included in the article's Creative Commons license, unless indicated otherwise in a credit line to the material. If material is not included in the article's Creative Commons license and your intended use is not permitted by statutory regulation or exceeds the permitted use, you will need to obtain permission directly from the copyright holder. To view a copy of this license, visit http://creativecommons.org/licenses/by/4.0/.

(c) The Author(s) 2019 